\title{
LINE LOAD APPLIED ALONG GENERATORS OF THIN-WALLED CIRCULAR CYLINDRICAL SHELLS OF FINITE LENGTH*
}

\author{
BY \\ N. J. HOFF, JOSEPH KEMPNER, AND FREDERICK V. POHLE \\ Polytechnic Institute of Brooklyn, Brooklyn, N. Y.
}

Summary. Donnell's differential equations of the thin circular cylindrical shell are integrated in the case when the loads are radial forces or circumferential moments distributed sinusoidally along a generator. Closed form expressions are obtained for the displacements, internal moments, and the membrane stresses. In addition, loads distributed uniformly along a segment of a generator and concentrated loads are discussed and radial forces are combined into a longitudinal moment.

1. Introduction. One of the most common elements of structures and machinery is the thin-walled circular cylindrical shell. When other elements are attached to it, forces and moments are likely to be transmitted to the shell across the areas of contact because of gravity and inertia effects, and in consequence of thermal expansion. These forces and moments can often be represented, with an accuracy sufficient for engineering purposes, as loads distributed along a short segment of a generator. For this reason it is of practical interest to investigate the deformations and the stresses of thin-walled circular cylindrical shells loaded along generators.

The problem of loads along generators was first solved by Finsterwalder [1] in 1932 in his investigation of the disturbance stresses arising at the free edge of a circular cylindrical shell-type roof structure. In his approximate theory the longitudinal moment $M_{x}$ and the torque $M_{x \varphi}$ in the shell were disregarded and a single partial differential equation in the circumferential moment $M_{\varphi}$ was derived. This approach was further simplified by Schorer [2] in 1935 whose differential equation in $M_{\varphi}$ consisted of only two terms. In the same year Dischinger [3] derived a rigorous solution. In order to reduce the large amount of work necessary to obtain numerical results from the rigorous theory, Aas Jakobsen [4] developed an iteration procedure in 1939 through which the equilibrium conditions could be expressed with sufficient accuracy in terms of the radial displacement $w$ alone. In 1941 he showed [5] how this approach could be used in the calculation of the effects of concentrated loads.

The concentrated load problem was attacked independently by S. W. Yuan [6] in 1946 who solved Donnell's single eighth order differential equation in $w$ for an indefinitely long shell. The surface loading was represented by a Fourier series in the circumferential direction and by a Fourier integral in the longitudinal direction. The method of images was then used to obtain a solution for the cylinder of finite length. In the same year Odqvist [7] gave a closed form solution for the deflections of finite cylinders subjected to sinusoidally distributed line loads and infinite series solutions for the deflections of

\footnotetext{
*Received Jan. 29, 1953. This work was performed under a consulting contract with the Knolls Atomic Power Laboratory, Schenectady, N. Y., operated by the General Electric Company for the United States Atomic Energy Commission. The authors are indebted to the company, to the AFC, and to Mr. Daniel R. Miller, the project supervisor, for their permission to publish this article.
} 
finite cylinders subjected to concentrated loads. These results were obtained from Schorer's equation.

Although the work of German and Scandinavian civil engineers seems to indicate that the moment $M_{x}$ and the torque $M_{x \varphi}$ are unimportant in the balance of forces and moments in a reasonably long shell-type roof subjected to distributed loads, the same conclusion need not necessarily hold when line loads or concentrated loads are applied to the structure. Indeed, in the experiments performed by Schoessow and Kooistra [8], in which forces and moments were transmitted to a shell by means of comparatively large-diameter pipes, the ratio of the maximum longitudinal bending stress to the maximum circumferential bending stress was found to be close to one-half. Therefore some doubt arises regarding the range of applicability of Schorer's equation. But Odqvist's paper, based on this equation, is the only one to present results from which the stresses in the shell can be obtained without extensive calculations. This situation prompted the development of the new solution given in this paper.

In the derivations that follow the stipulation of the conditions of equilibrium in the forms presented by Love [9], Flügge [10], Biezeno and Grammel [11], and Timoshenko [12] is replaced by the simplified statement proposed by Donnell [13] and recently recommended by Batdorf [14]. In addition to the eighth-order differential equation in the single dependent variable $w$, used and re-derived by Yuan, two fourth order equations are satisfied rigorously. The dependent variables are $u$ and $w$ in one equation, and $v$ and $w$ in the other; $w$ is the radial displacement, $u$ the longitudinal displacement, and $v$ the circumferential displacement. Closed form solutions are given for the displacement, moment, and membrane stress quantities arising from sinusoidally distributed line loads. Series of these solutions are capable of representing the effects of loads constant over segments of generators as well as those of concentrated loads.

2. Basic equations. In the absence of surface and body forces Donnell's [13] equilibrium conditions of an element of a thin-walled circular cylindrical shell can be stated in the following form:

$$
\begin{gathered}
\nabla^{8} w+4 K^{4} w_{. x x x x}=0, \\
\nabla^{4} u=\nu w_{. x x x}-w_{. x \varphi \varphi}, \\
\nabla^{4} v=(2+\nu) w_{. x x \varphi}+w_{. \varphi \varphi \varphi},
\end{gathered}
$$

where the subscripts following a comma indicate differentiation,

$$
4 K^{4}=12\left(1-\nu^{2}\right)(a / h)^{2}, \quad D=E h^{3} /\left[12\left(1-\nu^{2}\right)\right],
$$

and $\nabla^{2}$ is Laplace's operator

$$
\nabla^{2}=\left(\partial^{2} / \partial x^{2}\right)+\left(\partial^{2} / \partial \varphi^{2}\right) .
$$

The non-dimensional distances and displacements are defined by the equations

$$
x=\left(x^{*} / a\right), \quad u=\left(u^{*} / a\right), \quad v=\left(v^{*} / a\right), \quad w=\left(w^{*} / a\right),
$$

and $x^{*}$ is the distance measured in the axial direction along a generator, $a \varphi$ that measured around the circumference, and $u^{*}, v^{*}$, and $w^{*}$ are the displacements in the axial, circumferential, and radial directions, respectively, as shown in Fig. 1a. The remaining geometrical and physical quantities are $a$, the mean radius of the shell; $h$, the thickness of 
the shell; $L$, the length of the shell; $E$, Young's modulus of elasticity; and $\nu$, Poisson's ratio.

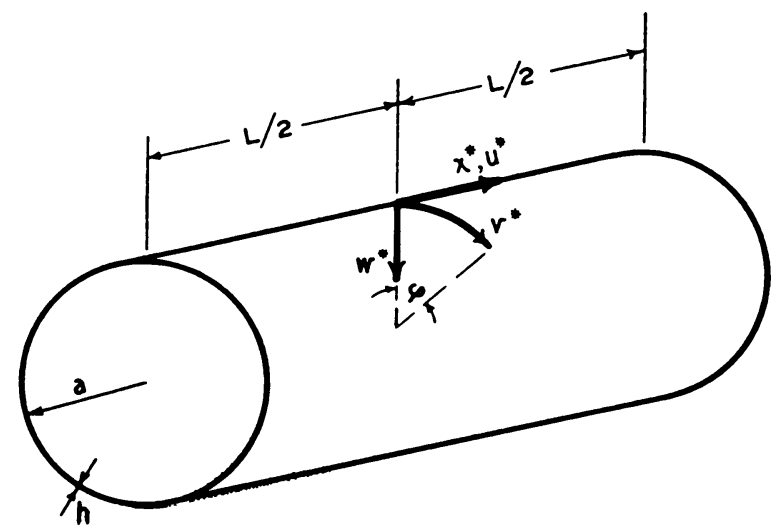

FIG. 1a.

In Donnell's approximation and with the present sign convention the strains $\epsilon$ and the curvatures $\kappa$ of the median surface of the shell are defined as

$$
\begin{aligned}
\epsilon_{x} & =u_{. x}, & \kappa_{x} & =(1 / a) w_{. x x}, \\
\epsilon_{\varphi} & =v_{. \varphi}-w, & \kappa_{\varphi} & =(1 / a) w_{. \varphi \varphi}, \\
\gamma_{x \varphi} & =u_{. \varphi}+v_{. x}, & \kappa_{x \varphi} & =(1 / a) w_{. x \varphi} .
\end{aligned}
$$

The membrane stresses can be given as

$$
\begin{aligned}
& \sigma_{x}= {\left[E /\left(1-\nu^{2}\right)\right]\left(\epsilon_{x}+\nu \epsilon_{\varphi}\right)=\left[E /\left(1-\nu^{2}\right)\right]\left(u_{, x}+\nu v_{. \varphi}-\nu w\right), } \\
& \sigma_{\varphi}= {\left[E /\left(1-\nu^{2}\right)\right]\left(\nu \epsilon_{x}+\epsilon_{\varphi}\right)=\left[E /\left(1-\nu^{2}\right)\right]\left(\nu u_{, x}+v_{. \varphi}-w\right), } \\
& \tau_{x \varphi}=[E / 2(1+\nu)] \gamma_{x \varphi}=[E / 2(1+\nu)]\left(u_{, \varphi}+v_{. x}\right) .
\end{aligned}
$$

The moment-resultants per unit length are indicated as right-hand vectors in Fig. lb. They are
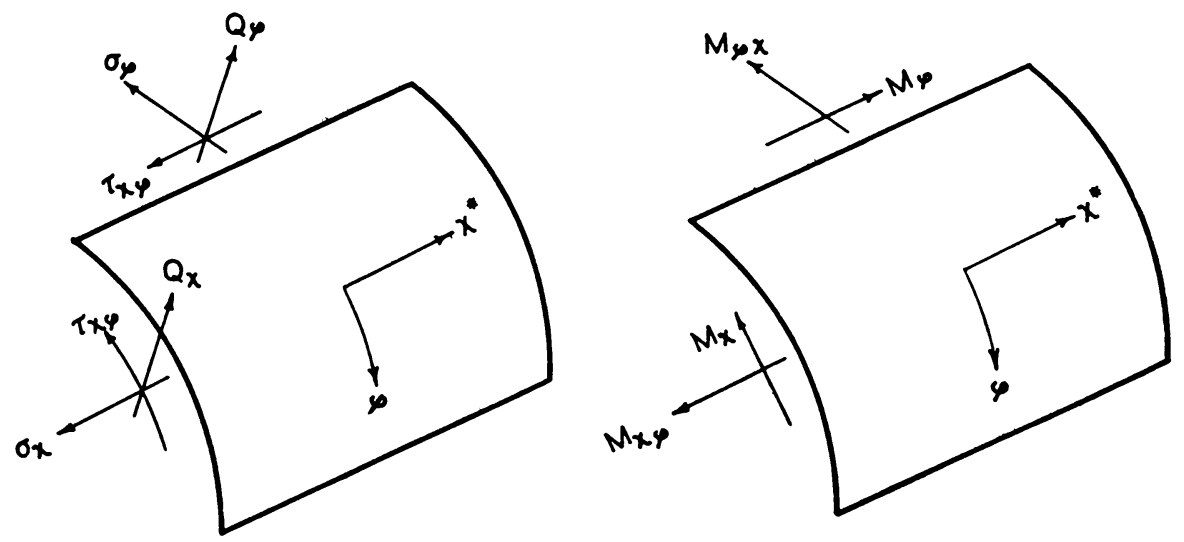

Fig. $1 b$. 


$$
\begin{aligned}
& M_{x}=-D\left(\kappa_{x}+\nu \kappa_{\varphi}\right)=-(D / a)\left(w_{. x x}+\nu w_{. \varphi \varphi}\right), \\
& M_{\varphi}=-D\left(\nu \kappa_{x}+\kappa_{\varphi}\right)=-(D / a)\left(w_{. \varphi \varphi}+\nu w_{. x x}\right), \\
& M_{x \varphi}=-M_{\varphi x}=(1-\nu) D \kappa_{x \varphi}=[(1-\nu) D / a] w_{, x \varphi} .
\end{aligned}
$$

The shear forces per unit length which accompany these moments and act in the radial direction are

$$
\begin{aligned}
& Q_{x}=(1 / a)\left(M_{x, x}+M_{\varphi x, \varphi}\right)=-\left(D / a^{2}\right)\left(w_{. x x x}+w_{. x \varphi \varphi}\right) \\
& Q_{\varphi}=(1 / a)\left(M_{\varphi, \varphi}-M_{x \varphi, x}\right)=-\left(D / a^{2}\right)\left(w_{. \varphi \varphi \varphi}+w_{, x x \varphi}\right)
\end{aligned}
$$

If the equivalent shear $(1 / a) M_{\varphi x . x}$ corresponding to the distributed torque is added to $Q_{\varphi}$ in agreement with Kirchhoff's suggestion (see, for instance, p. 90 of Reference 12), the total effective shear force per unit length is

$$
Q_{\varphi . \text { eff. }}=-\left(D / a^{2}\right)\left[w_{. \varphi \varphi \varphi}+(2-\nu) w_{, x x \varphi}\right]
$$

3. Sinusoidally distributed radial force symmetric with respect to $x=0$. It is assumed that the edges $x^{*}=(L / 2)$ are simply supported. This condition implies that the radial displacement $w$ vanishes and so do the axial membrane stress and the axial moment resultant, but not the membrane shear stress. If the expressions just given for these quantities are considered, the simple-support condition can be reduced to the following requirements:

$$
u_{. x}=v=w=w_{, x x}=0, \quad \text { when } \quad x= \pm(1 / 2)(L / a) .
$$

The load is applied along the generator $\varphi=0$ in the radial inward direction and is $2 V \cos n x$ with $n=m \pi(a / L)$ and $m$ an odd integer. With $-\pi \leq \varphi \leq \pi$, the solution is assumed as

$$
\begin{aligned}
& w_{\mathrm{tot}}=w(\varphi)+w(2 \pi-\varphi)+w(2 \pi+\varphi)+w(4 \pi-\varphi)+w(4 \pi+\varphi)+\cdots, \\
& u_{\mathrm{tot}}=u(\varphi)+u(2 \pi-\varphi)+u(2 \pi+\varphi)+u(4 \pi-\varphi)+u(4 \pi+\varphi)+\cdots, \\
& v_{\mathrm{tot}}=v(\varphi)-v(2 \pi-\varphi)+v(2 \pi+\varphi)-v(4 \pi-\varphi)+v(4 \pi+\varphi)-\cdots,
\end{aligned}
$$

where the dependence on $x$ is not shown but is understood, and

$$
\begin{aligned}
& w(\varphi)=A e^{p \varphi} \cos n x, \\
& u(\varphi)=B e^{p \varphi} \sin n x, \\
& v(\varphi)=C e^{p \varphi} \cos n x,
\end{aligned}
$$

where $A$ and $n$ are assumed real and $B, C$, and $p$ may be complex. These displacements can be imagined to be contributed by two fictitious sheets of width $L$, each beginning at $\varphi=0$ and winding around the actual shell indefinitely many times, one clockwise and the other counterclockwise. Only thase solutions will be considered in this analysis in which the real part of $p$ is negative.

It can be seen that the assumed deflections satisfy the boundary conditions at 
$x= \pm(1 / 2)(L / a)$. In the circumferential direction they are continuous everywhere except at $\varphi=0$. By a suitable choice of the conditions along the generator $\varphi=0$, continuity can be enforced there and the prescribed line load can be applied to the shell. Worked-out numerical examples have shown that in most cases the first term of the infinite series suffices for a satisfactory approximation to the deflections, and two terms are needed only infrequently.

The boundary conditions at $\varphi=0$ are:

(1) For reasons of symmetry

$$
w_{. \varphi}=0 \quad \text { when } \quad \varphi=0 .
$$

(2) To eliminate gaps caused by the circumferential displacements

$$
v=0 \quad \text { when } \quad \varphi=0 .
$$

(3) The shear stress $\tau_{x \varphi}$ acts in the same direction along the edges of the two sheets at $\varphi=0$. In the absence of a longitudinal line load, $\tau_{x \varphi}$ must vanish. Hence

$$
u_{. \varphi}+v_{. x}=0 \text { when } \varphi=0 .
$$

But $v_{, x}$ vanishes at $\varphi=0$ in consequence of (16). Therefore the requirement reduces to

$$
u_{. \varphi}=0 \text { when } \varphi=0 \text {. }
$$

(4) Finally the total effective shear force $Q_{\varphi_{\text {eft }}}$, given in (11), must be equal to one-half the applied radial load. However the second term in the brackets vanishes in consequence of (15) with the result that

$$
\left(D / a^{2}\right) w_{. \varphi \varphi \varphi}=V \cos n x .
$$

4. Enforcement of the differential equations. Substitution into (1) of the expression assumed for $w$ yields

$$
\left[\left(p^{2}-n^{2}\right)^{4}+4 K^{4} n^{4}\right] w=0
$$

which must be satisfied identically. Hence

$$
p^{2}=n^{2}+\sqrt{2}(-1)^{1 / 4} n K .
$$

If the fourth root of -1 is taken as $(\sqrt{2} / 2)(1-i)$ then

$$
p_{1}^{2}=n^{2}+(1-i) n K,
$$

and if $p_{1}$ is written as

$$
p_{1}=-\alpha_{1}+i \beta_{1},
$$

where $\alpha_{1}$ and $\beta_{1}$ are positive real numbers, one has

Finally

$$
\begin{gathered}
\alpha_{1}^{2}-\beta_{1}^{2}=n(n+K), \\
\alpha_{1} \beta_{1}=(1 / 2) n K, \\
\beta_{1}=(1 / 2)\left(n K / \alpha_{1}\right) .
\end{gathered}
$$

$$
\alpha_{1}^{2}=(n / 2)\left\{(n+K)+\left[(n+K)^{2}+K^{2}\right]^{1 / 2}\right\} .
$$


The square root is taken with the positive sign because $\alpha_{1}$ must be real by assumption.

When the choice is

$$
(-1)^{1 / 4}=(\sqrt{2} / 2)(-1-i),
$$

the value of $p$ is designated as $p_{2}$. The above equations are replaced by

and

$$
p_{2}=-\alpha_{2}+i \beta_{2},
$$

$$
\begin{gathered}
\alpha_{2}^{2}-\beta_{2}^{2}=n(n-K), \\
\alpha_{2} \beta_{2}=(1 / 2) n K, \\
\beta_{2}=(1 / 2)\left(n K / \alpha_{2}\right), \\
\alpha_{2}^{2}=(n / 2)\left\{(n-K)+\left[(n-K)^{2}+K^{2}\right]^{1 / 2}\right\} .
\end{gathered}
$$

The conjugate solutions, corresponding to the remaining two fourth roots of -1 , namely

$$
p_{3}=-\alpha_{1}-i \beta_{1}, \quad p_{4}=-\alpha_{2}-i \beta_{2}
$$

need not be discussed here in detail because they are governed by the same relationships as $p_{1}$ and $p_{2}$, respectively.

Of course the solutions for $\alpha_{1}$ and $\alpha_{2}$ of (20d) and (21d) can be negative as well as positive. The negative values are rejected because they lead to positive real parts of the exponent $p$ and thus yield displacements which increase without bounds as $\varphi$ increases.

If the deflections assumed in (14) for $u$ and $w$ are substituted in equilibrium condition (2) one obtains

$$
B\left(p^{2}-n^{2}\right)^{2}=A n\left(m^{2}+p^{2}\right) .
$$

Consequently

$$
B=A m^{2}\left[1 /\left(p^{2}-n^{2}\right)^{2}\right]+A n\left[p^{2} /\left(p^{2}-n^{2}\right)^{2}\right] .
$$

When $p=p_{1}$ one has

$$
\begin{gathered}
\left(p_{1}^{2}-n^{2}\right)^{2}=-i\left(2 K^{2} n^{2}\right), \quad 1 /\left(p_{1}^{2}-n^{2}\right)^{2}=+\left[i / 2 K^{2} n^{2}\right], \\
p_{1}^{2} /\left(p_{1}^{2}-n^{2}\right)^{2}=(1 / 2 K n)+i\left[(n+K) / 2 K^{2} n\right] .
\end{gathered}
$$

Equation (23) becomes

$$
B=\left(A / 2 K^{2}\right)\{K+i[(1+\nu) n+K]\} \text { when } p=p_{1} .
$$

When $p=p_{2}$ the equations following (23) become

$$
\begin{gathered}
\left(p_{2}^{2}-n^{2}\right)^{2}=i\left(2 K^{2} n^{2}\right), \quad 1 /\left(p_{2}^{2}-n^{2}\right)^{2}=-\left[i / 2 K^{2} n^{2}\right] \\
\left.p_{2}^{2} /\left(p_{2}^{2}-n^{2}\right)^{2}=-(1 / 2 K n)-i \mid(n-K) / 2 K^{2} n\right]
\end{gathered}
$$

and thus

$$
B=\left(A / 2 K^{2}\right)\{-K-i[(1+\nu) n-K]\} \quad \text { when } \quad p=p_{2} \text {. }
$$


Substitutions from (14a) and (14c) into (3) result in

$$
C\left(p^{2}-n^{2}\right)^{2}=A p\left[p^{2}-(2+\nu) n^{2}\right] .
$$

Manipulations similar to the ones shown lead to

$$
C=\left(A / 2 K^{2} n\right)\left\{-\alpha_{1} K+\beta_{1}[(1+\nu) n-K]+i\left\{\beta_{1} K+\alpha_{1}[(1+\nu) n-K]\right\}\right\}
$$

when $p=p_{1}$ and to

$$
C=\left(A / 2 K^{2} n\right)\left\{\alpha_{2} K-\beta_{2}[(1+\nu) n+K]+i\left\{-\beta_{2} K-\alpha_{2}[(1+\nu) n+K]\right\}\right\}
$$

when $p=p_{2}$.

Instead of making use of all the four exponents $p_{1}$ to $p_{4}$, it is more convenient to give the radial deflections $w$ in the following form:

$$
w=\left[\left(A_{1} R+A_{2} I\right) e^{p_{1} \varphi}+\left(A_{3} R+A_{4} I\right) e^{p_{2} \varphi}\right] \cos n x .
$$

Here the expressions in parentheses are operators with $R$ signifying the real part and $I$ the imaginary part of the complex function upon which the operation is performed. Equation (29) can be written explicitly as $w=\left[A_{1} e^{-\alpha_{1} \varphi} \cos \beta_{1} \varphi+A_{2} e^{-\alpha_{1} \varphi} \sin \beta_{1} \varphi+A_{3} e^{-\alpha_{2} \varphi} \cos \beta_{2} \varphi+A_{4} e^{-\alpha_{2} \varphi} \sin \beta_{2} \varphi\right] \cos (n x)$.

From (14b), (24), and (25) one obtains $u=\left(A_{1} R+A_{2} I\right)\left(1 / 2 K^{2}\right)\{K+i[(1+\nu) n+K]\} e^{-\alpha_{1} \varphi}\left(\cos \beta_{1} \varphi+i \sin \beta_{1} \varphi\right) \sin (n x)$ $+\left(A_{3} R+A_{4} I\right)\left(1 / 2 K^{2}\right)\{-K-i[(1+\nu) n-K]\} e^{-\alpha_{2} \varphi}\left(\cos \beta_{2} \varphi+i \sin \beta_{2} \varphi\right) \sin (n x)$.

When the operations indicated are performed, (31) becomes $u=\left(A_{1} / 2 K^{2}\right) e^{-\alpha_{1} \varphi}\left\{K \cos \beta_{1} \varphi-[(1+\nu) n+K] \sin \beta_{1} \varphi\right\} \sin n x$

$$
\begin{aligned}
& +\left(A_{2} / 2 K^{2}\right) e^{-\alpha_{2} \varphi}\left\{[(1+\nu) n+K] \cos \beta_{1} \varphi+K \sin \beta_{1} \varphi\right\} \sin n x \\
& +\left(A_{3} / 2 K^{2}\right) e^{-\alpha_{2} \varphi}\left\{-K \cos \beta_{2} \varphi+[(1+\nu) n-K] \sin \beta_{2} \varphi\right\} \sin n x \\
& +\left(A_{4} / 2 K^{2}\right) e^{-\alpha_{2} \varphi}\left\{-[(1+\nu) n-K] \cos \beta_{2} \varphi-K \sin \beta_{2} \varphi\right\} \sin n x .
\end{aligned}
$$

The circumferential displacement is found in a similar manner:

$$
\begin{aligned}
v=\left(A_{1} / 2 K^{2} n\right) e^{-\alpha_{1} \varphi}\{\{ & \left.-\alpha_{1} K+\beta_{1}[(1+\nu) n-K]\right\} \cos \beta_{1} \varphi \\
& \left.-\left\{\beta_{1} K+\alpha_{1}[(1+\nu) n-K]\right\} \sin \beta_{1} \varphi\right\}(\cos n x) \\
& +\left(A_{2} / 2 K^{2} n\right) e^{-\alpha_{1} \varphi}\left\{\left\{\beta_{1} K+\alpha_{1}[(1+\nu) n-K]\right\} \cos \beta_{1} \varphi\right. \\
& \left.+\left\{-\alpha_{1} K+\beta_{1}[(1+\nu) n-K]\right\} \sin \beta_{1} \varphi\right\}(\cos n x) \\
& +\left(A_{3} / 2 K^{2} n\right) e^{-\alpha_{2} \varphi}\left\{\left\{\alpha_{2} K-\beta_{2}[(1+\nu) n+K]\right\} \cos \beta_{2} \varphi\right. \\
& \left.+\left\{\beta_{2} K+\alpha_{2}[(1+\nu) n+K]\right\} \sin \beta_{2} \varphi\right\}(\cos n x) \\
& +\left(A_{4} / 2 K^{2} n\right) e^{-\alpha, \varphi}\left\{\left\{-\beta_{2} K-\alpha_{2}[(1+\nu) n+K]\right\} \cos \beta_{2} \varphi\right. \\
& \left.+\left\{\alpha_{2} K-\beta_{2}[(1+\nu) n+K]\right\} \sin \beta_{2} \varphi\right\}(\cos n x) .
\end{aligned}
$$


5. Enforcement of the boundary conditions at $\varphi=0$. From (29)

$$
w_{, \varphi}=\left[\left(A_{1} R+A_{2} I\right) p_{1} e^{\nu_{1} \varphi}+\left(A_{3} R+A_{4} I\right) p_{2} e^{\nu_{\varphi} \varphi}\right] \cos (n x) .
$$

At $\varphi=0$ the function $w_{. \varphi}$ must vanish. Hence the requirement contained in (15) leads to

$$
\left(A_{1} R+A_{2} I\right) p_{1}+\left(A_{3} R+A_{4} I\right) p_{2}=0 .
$$

This can also be written as

$$
-\alpha_{1} A_{1}+\beta_{1} A_{2}-\alpha_{2} A_{3}+\beta_{2} A_{4}=0 .
$$

From (16) and (33) one obtains

$$
\begin{aligned}
& \left\{-\alpha_{1} K+\beta_{1}[(1+\nu) n-K]\right\} A_{1}+\left\{\beta_{1} K+\alpha_{1}[(1+\nu) n-K]\right\} A_{2} \\
& \quad+\left\{\alpha_{2} K-\beta_{2}[(1+\nu) n+K]\right\} A_{3}+\left\{-\beta_{2} K-\alpha_{2}[(1+\nu) n+K]\right\} A_{4}=0 .
\end{aligned}
$$

To satisfy the conditions expressed by (17), $u_{.}$must be calculated:

$$
\begin{aligned}
u_{. \varphi}=\left(1 / 2 K^{2}\right)\left(A_{1} R+\right. & \left.A_{2} I\right)\{K+i[(1+\nu) n+K]\} p_{1} e^{p_{1} \varphi} \sin (n x) \\
& +\left(1 / 2 K^{2}\right)\left(A_{3} R+A_{4} I\right)\{-K-i[(1+\nu) n-K]\} p_{2} e^{p_{2} \varphi} \sin (n x) .
\end{aligned}
$$

Evaluation of this expression for $\varphi=0$ results in

$$
\begin{aligned}
& \left\{-\alpha_{1} K-\beta_{1}[(1+\nu) n+K]\right\} A_{1}+\left\{\beta_{1} K-\alpha_{1}[(1+\nu) n+K]\right\} A_{2} \\
& \quad+\left\{\alpha_{2} K+\beta_{2}[(1+\nu) n-K]\right\} A_{3}+\left\{-\beta_{2} K+\alpha_{2}[(1+\nu) n-K]\right\} A_{4}=0 .
\end{aligned}
$$

From these conditions $A_{1}, A_{2}$, and $A_{3}$ can be expressed in terms of $A_{4}$ :

$$
\begin{aligned}
& A_{1}=-\left(\frac{\alpha_{1}-\beta_{1}}{\alpha_{1}^{2}+\beta_{1}^{2}}\right)\left(\frac{\alpha_{2}^{2}+\beta_{2}^{2}}{\alpha_{2}-\beta_{2}}\right) A_{4}, \\
& A_{2}=\left(\frac{\alpha_{1}+\beta_{1}}{\alpha_{1}^{2}+\beta_{1}^{2}}\right)\left(\frac{\alpha_{2}^{2}+\beta_{2}^{2}}{\alpha_{2}-\beta_{2}}\right) A_{4}, \\
& A_{3}=\left(\frac{\alpha_{2}+\beta_{2}}{\alpha_{2}-\beta_{2}}\right) A_{4} .
\end{aligned}
$$

In the calculation of the shear, $w_{. \varphi \varphi \varphi}$ is needed. From (29) one obtains

$$
w_{. \varphi \varphi \varphi}=\left[\left(A_{1} R+A_{2} I\right) p_{1}^{3} e^{\nu_{2} \varphi}+\left(A_{3} R+A_{4} I\right) p_{2}^{3} e^{\nu_{1} \varphi}\right] \cos (n x) .
$$

At $\varphi=0$ this equation becomes

$$
w_{, \varphi \varphi \varphi}=\left[\left(A_{1} R+A_{2} I\right) p_{1}^{3}+\left(A_{3} R+A_{4} I\right) p_{2}^{2}\right] \cos (n x) .
$$

Evaluation of the operations indicated in (41) and substitution in (18) result in

$$
\begin{aligned}
V=-\left(D / a^{2}\right)\left\{\left(\alpha_{1}^{3}-3 \alpha_{1} \beta_{1}^{2}\right) A_{1}+\left(\beta_{1}^{3}-\right.\right. & \left.3 \beta_{1} \alpha_{1}^{2}\right) A_{2} \\
& \left.+\left(\alpha_{2}^{3}-3 \alpha_{2} \beta_{2}^{2}\right) A_{3}+\left(\beta_{2}^{3}-3 \beta_{2} \alpha_{2}^{2}\right) A_{4}\right\} .
\end{aligned}
$$


If the expressions given in (38), (39), and (40) are substituted into (42), manipulations yield

$$
V=4 K n\left(D / a^{2}\right)\left(\frac{\alpha_{2}^{2}+\beta_{2}^{2}}{\alpha_{2}-\beta_{2}}\right) A_{4} .
$$

This can be solved for $A_{4}$ :

$$
A_{4}=\frac{V}{D} \frac{a^{2}}{4 K n}\left(\frac{\alpha_{2}-\beta_{2}}{\alpha_{2}^{2}+\beta_{2}^{2}}\right) .
$$

The other three constants can also be expressed directly in terms of the applied load:

$$
\begin{aligned}
& A_{1}=-\frac{V}{D} \frac{a^{2}}{4 K n}\left(\frac{\alpha_{1}-\beta_{1}}{\alpha_{1}^{2}+\beta_{1}^{2}}\right), \\
& A_{2}=\frac{V}{D} \frac{a^{2}}{4 K n}\left(\frac{\alpha_{1}+\beta_{1}}{\alpha_{1}^{2}+\beta_{1}^{2}}\right), \\
& A_{3}=\frac{V}{D} \frac{a^{2}}{4 K n}\left(\frac{\alpha_{2}+\beta_{2}}{\alpha_{2}^{2}+\beta_{2}^{2}}\right) .
\end{aligned}
$$

The expressions for the displacements $u, v$, and $w$ are then as follows:

$$
\begin{aligned}
\left.u=\frac{V a^{2} \cdot\left\{\frac { e ^ { - \alpha _ { 1 } \varphi } } { \alpha _ { 1 } ^ { 2 } + \beta _ { 1 } ^ { 2 } } \left\{\left[2 \beta_{1} K\right.\right.\right.}{}+n(1+\nu)\left(\alpha_{1}+\beta_{1}\right)\right] \cos \beta_{1} \varphi \\
\left.+\left[2 \alpha_{1} K+n(1+\nu)\left(\alpha_{1}-\beta_{1}\right)\right] \sin \beta_{1} \varphi\right\} \\
+\frac{e^{-\alpha_{2} \varphi}}{\alpha_{2}^{2}+\beta_{2}^{2}}\left\{\left[-2 \beta_{2} K-n(1+\nu)\left(\alpha_{2}-\beta_{2}\right)\right] \cos \beta_{2} \varphi\right. \\
\left.\left.+\left[-2 \alpha_{2} K+n(1+\nu)\left(\alpha_{2}+\beta_{2}\right)\right] \sin \beta_{2} \varphi\right\}\right\} \sin (n x),
\end{aligned}
$$

$v=\frac{V a^{2}}{8 K^{3} n^{2} D}\left\{e^{-\alpha_{1} \varphi}\left\{n(1+\nu) \cos \beta_{1} \varphi+[n(1+\nu)-2 K] \sin \beta_{1} \varphi\right\}\right.$

$$
\left.+e^{-\alpha_{2} \varphi}\left\{-n(1+\nu) \cos \beta_{2} \varphi+[n(1+\nu)+2 K] \sin \beta_{2} \varphi\right\}\right\} \cos (n x),
$$$$
w=\frac{V a^{2}}{4 K n D}\left\{\frac{e^{-\alpha_{1} \varphi}}{\alpha_{1}^{2}+\beta_{1}^{2}}\left\{-\left(\alpha_{1}-\beta_{1}\right) \cos \beta_{1} \varphi+\left(\alpha_{1}+\beta_{1}\right) \sin \beta_{1} \varphi\right\}\right.
$$

$$
\left.+\frac{e^{-\alpha_{2} \varphi}}{\alpha_{2}^{2}+\beta_{2}^{2}}\left\{\left(\alpha_{2}+\beta_{2}\right) \cos \beta_{2} \varphi+\left(\alpha_{2}-\beta_{2}\right) \sin \beta_{2} \varphi\right\}\right\} \cos (n x) .
$$

6. Constant radial force over segment of generator. The results obtained can be used in the calculation of the displacements and stresses of cylinders subjected to symmetric radial forces of all kinds. For instance, when the inward radial load is stipulated as

$$
\left(P^{*} / 2 \delta\right) \text { when }\left|x^{*}\right| \leq \delta \text { and as } 0 \text { when }(L / 2) \geq\left|x^{*}\right|>\delta
$$


it can be represented by the Fourier series

$$
\sum_{m=1,3, \ldots}^{\infty} P^{*(m)} \cos (m \pi a x / L)
$$

with the coefficient $P^{*(m)}$ defined as

$$
P^{*(m)}=\left(2 P^{*} / m \pi \delta\right) \sin (m \pi \delta / L) \quad m=1,3, \cdots .
$$

The displacements $u, v$, and $w$ can then be calculated from (48), (49), and (50), respectively, if $V$ is replaced by $(1 / 2) P^{*(m)}$ and the resulting expressions are summed over $m$. Naturally the summation indicated in (13) must also be carried out if the first term alone does not give sufficient accuracy. However, this is seldom the case. The summation causes no difficulties because the right-hand members of (13) are geometric series.

If the coefficient $b_{m}$ is defined as

$$
b_{m}=\left(P^{*} a L_{i}^{\prime} 4 \pi^{2} D K \delta\right)\left(1 / m^{2}\right) \sin (m \pi \delta / L)
$$

substitutions and simplifications yield the following expressions for the $m$ th components of the moments and the membrane stresses:

$$
\begin{aligned}
M_{x}^{(m)} /\left[(-D / a) n b_{m} \cos n x\right]= & \frac{e^{-\alpha_{2} \varphi}}{\alpha_{1}^{2}+\beta_{1}^{2}}\left\{\left[-2 \nu K \alpha_{1}+n(1-\nu)\left(\alpha_{1}-\beta_{1}\right)\right] \cos \beta_{1} \varphi\right. \\
& \left.+\left[2 \nu K \beta_{1}-n(1-\nu)\left(\alpha_{1}+\beta_{1}\right)\right] \sin \beta_{1} \varphi\right\} \\
& +\frac{e^{-\alpha_{2} \varphi}}{\alpha_{2}^{2}+\beta_{2}^{2}}\left\{\left[-2 \nu K \alpha_{2}-n(1-\nu)\left(\alpha_{2}+\beta_{2}\right)\right] \cos \beta_{2 \varphi}\right. \\
& \left.+\left[2 \nu K \beta_{2}-n(1-\nu)\left(\alpha_{2}-\beta_{2}\right)\right] \sin \beta_{2} \varphi\right\}
\end{aligned}
$$

$M_{\varphi}^{(m)} /\left[(-D / a) n b_{m} \cos n x\right]=\frac{e^{-\alpha_{1} \varphi}}{\alpha_{1}^{2}+\beta_{1}^{2}}\left\{\left[-2 K \alpha_{1}-n(1-\nu)\left(\alpha_{1}-\beta_{1}\right)\right] \cos \beta_{1} \varphi\right.$

$$
\begin{aligned}
& \left.+\left[2 K \beta_{1}+n(1-\nu)\left(\alpha_{1}+\beta_{1}\right)\right] \sin \beta_{1} \varphi\right\} \\
& +\frac{e^{-\alpha_{2} \varphi}}{\alpha_{2}^{2}+\beta_{2}^{2}}\left\{\left[-2 K \alpha_{2}+n(1-\nu)\left(\alpha_{2}+\beta_{2}\right)\right] \cos \beta_{2 \varphi}\right. \\
& \left.+\left[2 K \beta_{2}+n(1-\nu)\left(\alpha_{2}-\beta_{2}\right)\right] \sin \beta_{2} \varphi\right\},
\end{aligned}
$$

$. I_{x \varphi}^{(m)} /\left[(D / a)(1-\nu) n b_{m} \sin n x\right]=e^{-\alpha_{1} \varphi}\left[-\cos \beta_{1} \varphi+\sin \beta_{1} \varphi\right]$

$$
+e^{-\alpha_{2} \varphi}\left[\cos \beta_{2} \varphi+\sin \beta_{2} \varphi\right]
$$

$\sigma_{x}^{(m)} /\left[\left(E / 2 K^{2}\right) n b_{m} \cos n x\right]$

$$
\begin{aligned}
= & \frac{e^{-\alpha_{1} \varphi}}{\alpha_{1}^{2}+\beta_{1}^{2}}\left\{\left[n \alpha_{1}+(n+2 K) \beta_{1}\right] \cos \beta_{1} \varphi+\left[(n+2 K) \alpha_{1}-n \beta_{1}\right] \sin \beta_{1} \varphi\right\} \\
& +\frac{e^{-\alpha_{2} \varphi}}{\alpha_{2}^{2}+\beta_{2}^{2}}\left\{\left[-n \alpha_{2}+(n-2 K) \beta_{2}\right] \cos \beta_{2} \varphi+\left[(n-2 K) \alpha_{2}+n \beta_{2}\right] \sin \beta_{2} \varphi\right\},
\end{aligned}
$$




$$
\begin{aligned}
\sigma_{\varphi}^{(m)} /\left[\left(E / 2 K^{2}\right) n^{2} b_{m} \cos n x\right]= & \frac{e^{-\alpha_{1} \varphi}}{\alpha_{2}^{2}+\beta_{2}^{2}}\left\{-\left(\alpha_{1}+\beta_{1}\right) \cos \beta_{1} \varphi+\left(-\alpha_{1}+\beta_{1}\right) \sin \beta_{1} \varphi\right\} \\
& +\frac{e^{-\alpha_{2} \varphi}}{\alpha_{2}^{2}+\beta_{2}^{2}}\left\{\left(\alpha_{2}-\beta_{2}\right) \cos \beta_{2} \varphi-\left(\alpha_{2}+\beta_{2}\right) \sin \beta_{2} \varphi\right\}
\end{aligned}
$$

$\tau_{x \varphi}^{(m)} /\left[\left(E / 2 K^{2}\right) n b_{m} \sin n x\right]=-e^{-\alpha_{1} \varphi}\left\{\cos \beta_{1} \varphi+\sin \beta_{1} \varphi\right\}$

$$
+e^{-\alpha_{2} \varphi}\left\{\cos \beta_{2} \varphi-\sin \beta_{2} \varphi\right\} .
$$

When the load is concentrated at $x=0$, the distance $\delta$ approaches zero. In that case

and

$$
P^{(m)}=\left(2 P^{*} / L\right),
$$

$$
b_{m}=\left(P^{*} a L / 4 \pi D K\right)(1 / m) .
$$

7. Sinusoidally distributed radial force antisymmetric with respect to $x=0$. The load is applied along the generator $\varphi=0$ in the radial inward direction and is $2 V \sin n x$ with $n=m \pi(a / L)$ and $m$ an even integer. The solution is assumed in the form of the series (13) with

$$
\begin{aligned}
& w=A e^{p \varphi} \sin n x, \\
& u=B e^{p \varphi} \cos n x, \\
& v=C e^{p \varphi} \sin n x,
\end{aligned}
$$

where again $A$ and $n$ are assumed real while $B, C$, and $p$ may be complex. The boundary conditions at $x= \pm(1 / 2)(L / a)$ and at $\varphi=0$ remain unchanged except that

$$
\left(D / a^{2}\right) w_{. \varphi \varphi \varphi}=V \sin n x .
$$

Solution of this problem leads to the same expressions for the constants $A_{1}$ to $A_{4}$ as obtained earlier and presented in (44) to (47). Similarly the expressions given in (49) and (50) for the displacements $v$ and $w$ are unchanged except for the replacement of the factors $(\cos n x)$ by $(\sin n x)$; also in expression (48) for $u$ the factor ( $\sin n x$ ) must be replaced by $(-\cos n x)$.

8. Loading by longitudinal moment. When the radial inward load along the generator $\varphi=0$ is stipulated as

$$
\begin{array}{ccrl}
M_{x}^{*} / \delta^{2} & \text { when } & 0 & \leq x^{*} \leq \delta, \\
-M_{x}^{*} / \delta^{2} & \text { when } & -\delta & \leq x^{*} \leq 0, \\
0 & \text { when } & (L / 2) & \geq\left|x^{*}\right|>\delta,
\end{array}
$$

where $M_{x}^{*}$ is the applied longitudinal moment to which the line loading is statically equivalent, the loading can be represented by the Fourier series

$$
\sum_{m=2,4}^{\infty} \ldots M_{x}^{*(m)} \sin (m \pi a x / L)
$$


The coefficient $M_{x}^{*(m)}$ is defined as

$$
M_{x}^{*(m)}=\left(4 M I_{x}^{*} / m \pi \delta^{2}\right)[1-\cos (m \pi \delta / L)] \quad m=2,4, \cdots .
$$

The deflections $u, v$, and $w$ can be calculated from (48), (49), and (50), respectively, if $V$ is replaced by $(1 / 2) M_{x}^{*(m)}$, the changes in sign and in the trigonometric expressions stated in the preceding article are carried out, and the resulting expressions are summed over $m$. Naturally the summation indicated in (13) must also be carried out if the first term alone does not give sufficient accuracy. However, this is seldom the case.

If the coefficient $b_{m}$ is now defined as

$$
b_{m}=\left(M_{x}^{*} a L / 2 \pi^{2} D K \delta^{2}\right)\left(1 / m^{2}\right)[1-\cos (m \pi \delta / L)] \quad m=2,4, \cdots
$$

the $m$ th components of the moments and of the membrane stresses are again given by equations (54) to (59) provided all factors $(\cos n x)$ are replaced by $(\sin n x)$ and all factors $(\sin n x)$ by $(-\cos n x)$.

When the moment is concentrated at $x=0$, the distance $\delta$ approaches zero. In that case

and

$$
M_{x}^{(m)}=\left(2 \pi M_{x}^{*} / L^{2}\right) m
$$

$$
b_{m}=\left(M_{x}^{*} / 4 D K\right)(a / L) .
$$

9. Sinusoidally distributed circumferential moment symmetric with respect to $x=0$. This problem differs from the symmetric sinusoidally distributed force problem only in the boundary conditions at $\varphi=0$. The solution is assumed in the form

$$
\begin{aligned}
w_{\mathrm{tot}}=w(\varphi)-w(2 \pi-\varphi)+w(2 \pi+\varphi)-w(4 \pi-\varphi)+w(4 \pi+\varphi)+\cdots, \\
u_{\mathrm{tot}}=u(\varphi)-u(2 \pi-\varphi)+u(2 \pi+\varphi)-u(4 \pi-\varphi)+u(4 \pi+\varphi)-\cdots, \\
v_{\mathrm{tot}}=v(\varphi)+v(2 \pi-\varphi)+v(2 \pi+\varphi)+v(4 \pi-\varphi)+v(4 \pi+\varphi)+\cdots, \\
w(\varphi)=A e^{p \varphi} \cos n x, \\
u(\varphi)=B e^{p \varphi} \sin n x, \\
v(\varphi)=C e^{p \varphi} \cos n x,
\end{aligned}
$$

where the dependence on $x$ is not shown but is understood, and $A$ and $n$ are assumed real while $B, C$, and $p$ may be complex.

Along the generator $\varphi=0$ an external moment $2 M \cos n x$ is applied with $n=m \pi(a / L)$ and $m$ an odd integer. The boundary conditions are

$$
\begin{gathered}
w=u=\sigma_{\varphi}=0 \quad \text { when } \quad \varphi=0 \\
M_{\varphi 0}=M \cos (\max / L)
\end{gathered}
$$

and $M_{\varphi 0}$ is the internal circumferential moment per unit length of the shell along the cut at $\varphi=0$.

It follows from (71), (31), (32), and (33) that

$$
\begin{gathered}
A_{1}+A_{3}=0 \\
2 A_{1} K+A_{2}[(1+\nu) n+K]-A_{4}[(1+\nu) n-K]=0 \\
2 A_{1} \nu K+A_{2}[(1+\nu) n+K]-A_{4}[(1+\nu) n-\nu K]=0 .
\end{gathered}
$$


Solution of (73) to (75) yields

$$
A_{4}=A_{3}=A_{2}=-A_{1} \text {. }
$$

The fourth boundary condition (72) along $\varphi=0$ gives

$$
A_{1}=-(1 / 4 \pi)(M L / K D)(1 / m) \text {. }
$$

Hence the displacements can be written as

$w=-(1 / 4 \pi)(M L / K D)(1 / m)\left[e^{-\alpha_{1} \varphi}\left(\cos \beta_{1} \varphi-\sin \beta_{1} \varphi\right)\right.$

$$
\left.-e^{-\alpha_{2} \varphi}\left(\cos \beta_{2} \varphi+\sin \beta_{2} \varphi\right)\right] \cos n x,
$$

$u=(1 / 8 \pi)\left(M L / K^{3} D\right)(1 / m)\left\{e^{-\alpha_{1} \varphi}\left\{(1+\nu) n \cos \beta_{1} \varphi+[(1+\nu) n+2 K] \sin \beta_{1} \varphi\right\}\right.$

$$
\left.-e^{-\alpha_{2} \varphi}\left\{(1+\nu) n \cos \beta_{2} \varphi-[(1+\nu) n-2 K] \sin \beta_{2} \varphi\right\}\right\} \sin n x,
$$

$v=(1 / 8 \pi)\left(M L / K_{-}^{3} D\right)(1 / m n)\left\{e^{-\alpha_{1} \varphi}\left\{\left[(1+\nu) n\left(\alpha_{1}-\beta_{1}\right)+2 \beta_{1} K\right] \cos \beta_{1} \varphi\right.\right.$

$$
\begin{aligned}
& \left.+\left[(1+\nu) n\left(\alpha_{1}+\beta_{1}\right)-2 \alpha_{1} K\right] \sin \beta_{1} \varphi\right\} \\
& -e^{-\alpha_{2} \varphi}\left\{\left[(1+\nu) n\left(\alpha_{2}+\beta_{2}\right)+2 \beta_{2} K\right] \cos \beta_{2} \varphi\right. \\
& \left.\left.-\left[(1+\nu) n\left(\alpha_{2}-\beta_{2}\right)+2 \alpha_{2} K\right] \sin \beta_{2} \varphi\right\}\right\} \cos n x,
\end{aligned}
$$

10. Constant circumferential moment over segment of generator. When the external circumferential moment is distributed according to the rule

$$
\begin{array}{ccrl}
M_{\phi}^{*} / 2 \delta & \text { when } & & \left|x^{*}\right| \leq \delta \\
0 & \text { when } & (L / 2) \geq\left|x^{*}\right|>\delta
\end{array}
$$

it can be represented by the Fourier series

$$
\sum_{m=1,3, \ldots}^{\infty} M_{\varphi}^{*(m)} \cos (m \pi a x / L)
$$

where the coefficient $M_{\varphi}^{*(m)}$ is

$$
M_{\varphi}^{*(m)}=\left(2 M_{\varphi}^{*} / \pi \delta\right)(1 / m) \sin (m \pi \delta / L) .
$$

The displacements $w, u$, and $v$ can be calculated from (78), (79), and (80) if $M$ is replaced by $(1 / 2) M_{\varphi}^{*(m)}$ and the resulting expressions are summed over $m$. Naturally the summation indicated in (69) must also be carried out if the first term alone does not give sufficient accuracy. However, this is seldom the case.

If the coefficient $b_{m}$ is defined as

$$
b_{m}=-\left(M_{\varphi}^{*} / 4 \pi^{2}\right)(L / D K \delta)\left(1 / m^{2}\right) \sin (m \pi \delta / L),
$$

the moments and the membrane stresses in the shell can be given in the form $\left.M_{z}^{(m)} / \mathrm{l}(-D / a) b_{m} n \cos n x\right]=e^{-\alpha_{2} \varphi}\left\{[-(1-\nu) n+2 \nu K] \cos \beta_{1} \varphi+(1-\nu) n \sin \beta_{1} \varphi\right\}$

$$
+e^{-\alpha, \varphi}\left\{\left[(1-\nu) n+{ }_{\omega \nu} K\right] \cos \beta_{2}^{2} \varphi+(1-\nu) n \sin \beta_{2} \varphi\right\},
$$


$M_{\varphi}^{(m)} /\left[(-D / a) b_{m} n \cos n x\right]=e^{-\alpha_{1} \varphi}\left\{[(1-\nu) n+2 K] \cos \beta_{1} \varphi-(1-\nu) n \sin \beta_{1} \varphi\right\}$

$$
+e^{-\alpha_{2} \varphi}\left\{[-(1-\nu) n+2 K] \cos \beta_{2} \varphi-(1-\nu) n \sin \beta_{2} \varphi\right\},
$$

$M_{x \varphi}^{(m)} /\left[(-D / a)(1-\nu) b_{m} n \sin n x\right]=e^{-\alpha_{1} \varphi}\left\{-\left(\alpha_{1}+\beta_{1}\right) \cos \beta_{1} \varphi+\left(\alpha_{1}-\beta_{1}\right) \sin \beta_{1} \varphi\right\}$

$$
+e^{-\alpha_{2} \varphi}\left\{\left(\alpha_{2}-\beta_{2}\right) \cos \beta_{2} \varphi+\left(\alpha_{2}+\beta_{2}\right) \sin \beta_{2} \varphi\right\},
$$

$\sigma_{x}^{(m)} /\left[\left(E / 2 K^{2}\right) b_{m} n \cos n x\right]=-e^{-\alpha_{1} \varphi}\left\{n \cos \beta_{1} \varphi+(n+2 K) \sin \beta_{1} \varphi\right\}$

$$
+e^{-\alpha_{2} \varphi}\left\{n \cos \beta_{2 \varphi}-(n-2 K) \sin \beta_{2} \varphi\right\},
$$

$\left.\sigma_{\varphi}^{(m)} /\left[E / 2 K^{2}\right) b_{m} n^{2} \cos n x\right]=e^{-\alpha_{2} \varphi}\left\{\cos \beta_{1} \varphi+\sin \beta_{1} \varphi\right\}$

$$
+e^{-\alpha_{s} \varphi}\left\{-\cos \beta_{2} \varphi+\sin \beta_{2} \varphi\right\},
$$

$\tau_{x \varphi}^{(m)} /\left[\left(E / 2 K^{2}\right) b_{m} n \sin n x\right]=e^{-\alpha_{1} \varphi}\left\{\left(\alpha_{1}-\beta_{1}\right) \cos \beta_{1} \varphi+\left(\alpha_{1}+\beta_{1}\right) \sin \beta_{1} \varphi\right\}$

$$
+e^{-\alpha_{2} \varphi}\left\{-\left(\alpha_{2}+\beta_{2}\right) \cos \beta_{2} \varphi+\left(\alpha_{2}-\beta_{2}\right) \sin \beta_{2} \varphi\right\} \text {. }
$$

When the moment is concentrated at $x=0$, the distance $\delta$ approaches zero. In that case

and

$$
M_{\varphi}^{(m)}=\left(2 M_{\varphi}^{*} / L\right)
$$

$$
b_{m}=-\left(M_{4}^{*} / 4 \pi D K\right)(1 / m) .
$$

11. Convergence. When $\delta$ is finite in the series representing the loads, all the displacements, moments, and stresses converge. This is true even when $\delta=0, x \neq 0$, and the load is a concentrated radial force $P^{*}$. In this case, when $m$ is sufficiently large, the quantities of practical interest behave at $\varphi=0$ in the following manner:

$$
\begin{array}{cc}
w \sim\left(1 / m^{3}\right) \cos (m \pi a x / L), & w_{, x} \sim\left(1 / m^{2}\right) \sin (m \pi a x / L), \\
M_{x} \sim(1 / m) \cos (m \pi a x / L), & M_{\varphi} \sim(1 / m) \cos (m \pi a x / L), \\
\sigma_{x} \sim\left(1 / m^{3}\right) \cos (m \pi a x / L), & \sigma_{\varphi} \sim\left(1 / m^{3}\right) \cos (m \pi a x / L), \\
\tau_{x \varphi}=M_{x \varphi}=0 .
\end{array}
$$

When $\varphi=0$ and $x=0$, the series for $M_{x}$ and $M_{\varphi}$ diverge, but the remaining series converge.

When the load is a concentrated longitudinal moment at $x=0, \varphi=0$, the behavior at $\varphi=0$ for large values of $m$ is:

$$
\begin{aligned}
& w \sim\left(1 / m^{2}\right) \sin (\max / L), w_{, x} \sim(1 / m) \cos (\max / L), \\
& M_{x} \sim \sin (\max / L), M_{\varphi} \sim \sin (m \pi a x / L), \\
& \sigma_{x} \sim\left(1 / m^{2}\right) \sin (\max / L), \sigma_{\varphi} \sim\left(1 / m^{2}\right) \sin (\operatorname{maax} / L), \\
& \tau_{x \varphi}=M_{x \varphi}=0
\end{aligned}
$$


The displacements and the membrane stresses again converge, hut the moments $M_{x}$ and $M_{\varphi}$ are giren by divergent series representing zero when $x \neq 0$ and an indefinitely large quantity when $x=0$. The series converge for all values of $\varphi \neq 0$.

Finally, when the load is a concentrated circumferential moment at $\varphi=0, x=0$, one obtains at $\varphi=0$ for sufficiently large $m$ :

$$
\begin{gathered}
w=w_{, x}=\sigma_{x}=\sigma_{\varphi}=0, \\
M_{x} \sim \cos (\max / L), \quad M_{\varphi} \sim \cos \left(\max ^{\prime}{ }^{\prime} L\right), \\
M_{x \varphi} \sim \sin \left(\max x^{\prime} L\right), \quad \tau_{x \varphi} \sim\left(1 / m^{2}\right) \sin \left(\max x^{\prime} L\right) .
\end{gathered}
$$

Here again the series for the moments diverge in consequence of the representation of the load by a divergent series. The series for the stresses converge, and all the quantities are represented by convergent series when $\varphi \neq 0$.

\section{REFERENCES}

1. Ulrich Finsterwalder, Die Theorie der zylindrischen Schalengewölbe System Zeiss-Dywidag und ihre Anwendung auf die Grossmarkthalle Budapest, Publications Internat. Assoc. Bridge and Structural Engg., vol. 1, p. 127, Zürich, 1932.

2. Herman Schorer, Line load action on thin cylindrical shells, Proc. ASCE, 61, 281 (1935).

3. Fr. Dischinger, Die strenge Theorie der Kreiszylinderschale in ihrer Anwendung auf die Zeiss-DywidagSchalen, Beton und Eisen, 34, 257, 283, 392 (1935).

4. A. Aas Jakobsen, Über das Randstörungsproblem an Kreiszylinderschalen, Bauingenieur, 20, 394 (1939).

5. A. Aas Jakobsen, Einzellasten auf Kreiszylinderschalen, Bauingenieur, 22, 343 (1941).

6. Shao Wen Yuan, Thin cylindrical shells subjected to concentrated loads, Q. Appl. Math., 4, 13 (1946).

7. F. K. G. Odqvist, Action of forces and moments symmetrically distributed along a generatrix of a thin cylindrical shell, J. Appl. Mech., 13, A-106 (1946).

8. G. J. Schoessow and L. F. Kooistra, Stresses in a cylindrical shell due to nozzle or pipe connection, J. Appl. Mech., 12, A-107 (1945).

9. A. E. H. Love, A treatise on the mathematical theory of elasticity, Fourth Edition, Dover Publications, New York, 1944, p. $5+5$.

10. Wilhelm Flügge, Statik und Dynamik der Schalen, Julius Springer, Berlin, 1934, p. 118.

11. C. B. Biezeno and R. Grammel, Technische Dynamik, Julius Springer, Berlin, 1939, p. 469.

12. S. Timoshenko, Theory of plates and shells, McGraw-Hill Book Co., New York, 1940, p. 440.

13. L. H. Donnell, Stability of thin-walled tubes under torsion, NACA Techn. Rep. No. 479, Washington, D. C., 1933.

14. S. B. Batdorf, A simplified method of elastic stability analysis for thin cylindrical shells, NACA Techn. Rep. No. 874, Washington, D. C., 1947. 